\title{
La déprofessionnalisation: un terme neutre pour un constat désolant
}

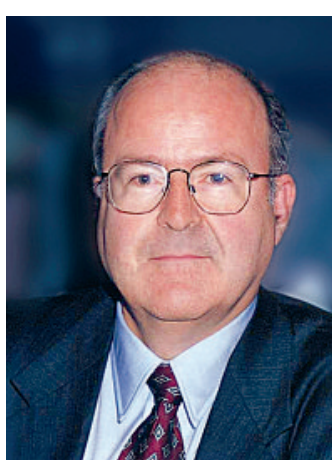

Werner Bauer
Le professeur Paul Unschuld est pharmacologue, sinologue, directeur émérite de l'Institut universitaire d'histoire de la médecine à Munich et directeur actuel du Horst-Görtz-Stiftungsinstituts de l'hôpital de la Charité à Berlin et fin observateur des développements dans le domaine de la santé.

Ses observations, publiées dans les livres (uniquement disponibles en allemand) «Der Arzt als Fremdling in der Medizin» [1] et "Ware Gesundheit» [2], n'ont cependant rien de réjouissant pour le corps médical. Les réflexions ci-dessous s'appuient sur des extraits de son analyse.

Unschuld part du principe qu'on reconnaît une profession libérale à trois paramètres:

Premièrement, les membres d'un groupe professionnel créent leur propre savoir. Deuxièmement, ils décident en toute indépendance comment l'utiliser et, troisièmement, ils peuvent décider quelle rémunération ils souhaitent obtenir pour leur activité professionnelle.

Il va de soi que cette indépendance n'a rien d'absolu, mais le corps médical a néanmoins réussi, au cours des siècles, à passer de la corporation des chirurgiens-barbiers et médecins itinérants à un véritable corps de métier qui, grâce à la recherche scientifique, à la bonne qualité de ses soins et à l'acquisition d'une position reconnue au sein de la société et dans le domaine de la santé, s'est fortement rapproché des paramètres précités.

Si par contre nous observons les changements auxquels est soumise la position du corps médical, depuis un certain temps, nous pouvons constater que l'autodétermination de notre profession est de plus en plus remise en cause et qu'elle a tendance à s'effilocher.

On me rétorquera peut-être qu'il y a aussi des avantages à ce que le temps des médecins-directeurs tout puissants ayant seuls le pouvoir de décision sur leur clinique soit révolu et qu'il est bon que les soignants et l'administration soient aussi inclus dans les processus de décision.

Ces mutations ont cependant pris une telle ampleur, qu'elles vont au-delà de la simple modification des hiérarchies et des règles de déontologie dépassées. Les débats politiques au sujet de la santé, les discussions sur les tarifs et les prestations, les questions ayant trait à l'introduction de nouveaux modèles (DRG), la promulgation de nouvelles lois et ordonnances ou la mise en œuvre d'adaptations structurelles dans les hôpitaux sont autant de domaines où le corps médical perd en influence. De plus en plus souvent, les médecins ne peuvent que prendre acte des décisions prises et les appliquer sans avoir eu leur mot à dire sur la question. Le dernier exemple en date concerne la révision par les autorités fédérales des tarifs de laboratoire.

Pour Paul Unschuld - qui qualifie ces mutations de déprofessionnalisation - il s'agit des prémices d'une tendance et les tendances sont, on le sait, difficilement influençables.

Unschuld cite un manager de la santé allemand lequel déclare que les médecins croient bénéficier d'une liberté artistique dans l'exercice de leur profession mais que, au vu de la réalité, ils traversent en fait une crise de sens dont ils ne pourraient se sortir même s'ils obtenaient une hausse de salaire de $30 \%$. Il constate ensuite que l'époque de la corporation de médecins touche à sa fin; après avoir été l'essence même du système de santé, le médecin joue parfois encore un rôle important dans certains domaines mais plutôt subalterne dans d'autres, déclassé dans un engrenage complexe principalement mis en mouvement par des critères économiques.

Puis il ajoute que le corps médical est en passe de devenir un groupe qui exécute ce que d'autres groupes sociaux ont décidé. Cette évolution ne s'explique que partiellement par des contraintes externes. Parfois, on ne peut s'empêcher de penser qu'une part grandissante de médecins manque d'aspiration pour embrasser une carrière indépendante et faire preuve de responsabilité individuelle.

Selon l'analyse d'Unschuld, les pharmaciens ne sont pas en reste, car ils sont eux aussi en voie de déprofessionnalisation. En effet, leur formation chèrement acquise n'est plus requise dans la vente de médicaments sans ordonnance, les conseils personnalisés sont remplacés par la publicité et la question de la tolérance médicamenteuse se règle sans problèmes et sans eux, via une carte de patient informatisée.

La nouvelle génération de médecins, qui n'aura rien connu d'autre, se satisfera-t-elle bon an mal an d'une activité «déprofessionnalisée» ou le corps médical se réveillera-t-il pour intervenir et se faire entendre de manière aussi efficace que compétente dans le domaine politique et économique, tant et si bien qu'il devra être pris au sérieux et consulté lors des prises de décisions? Une question à laquelle le Prof. Unschuld n'est pas non plus en mesure de répondre.

Les tendances sont certes puissantes, mais il y a toujours un retour de manivelle et ce d'autant plus si on essaie d'inverser la tendance!

Werner Bauer* de la European Federation of Internal Medicine (EFIM). 